Review

\title{
The role of the UPS in cystic fibrosis
}

\section{Emma L Turnbull, Meredith FN Rosser and Douglas M Cyr*}

Address: Department of Cell and Developmental Biology, 526 Taylor Hall, Mason Farm Road, UNC-Chapel Hill School of Medicine, University of North Carolina, Chapel Hill, North Carolina 27599, USA

Email: Emma L Turnbull - eturnbul@email.unc.edu; Meredith FN Rosser - mrosser@email.unc.edu; Douglas M Cyr* -dmcyr@med.unc.edu

* Corresponding author

Published: 22 November 2007

BMC Biochemistry 2007, 8(SuppI I):SII doi:I0.II86/I47I-209I-8-SI-SII

This article is available from: http://www.biomedcentral.com/I47I-209I/8/SI/SI I

(c) 2007 Turnbull et al.; licensee BioMed Central Ltd.

This is an open access article distributed under the terms of the Creative Commons Attribution License (http://creativecommons.org/licenses/by/2.0), which permits unrestricted use, distribution, and reproduction in any medium, provided the original work is properly cited.

\begin{abstract}
$\mathrm{CF}$ is an inherited autosomal recessive disease whose lethality arises from malfunction of CFTR, a single chloride $(\mathrm{Cl})$ ion channel protein. CF patients harbor mutations in the CFTR gene that lead to misfolding of the resulting CFTR protein, rendering it inactive and mislocalized. Hundreds of CFrelated mutations have been identified, many of which abrogate CFTR folding in the endoplasmic reticulum (ER). More than $70 \%$ of patients harbor the $\triangle$ F508 CFTR mutation that causes misfolding of the CFTR proteins. Consequently, mutant CFTR is unable to reach the apical plasma membrane of epithelial cells that line the lungs and gut, and is instead targeted for degradation by the UPS. Proteins located in both the cytoplasm and ER membrane are believed to identify misfolded CFTR for UPS-mediated degradation. The aberrantly folded CFTR protein then undergoes polyubiquitylation, carried out by an EI-E2-E3 ubiquitin ligase system, leading to degradation by the $26 \mathrm{~S}$ proteasome. This ubiquitin-dependent loss of misfolded CFTR protein can be inhibited by the application of 'corrector' drugs that aid CFTR folding, shielding it from the UPS machinery. Corrector molecules elevate cellular CFTR protein levels by protecting the protein from degradation and aiding folding, promoting its maturation and localization to the apical plasma membrane. Combinatory application of corrector drugs with activator molecules that enhance CFTR Cl- ion channel activity offers significant potential for treatment of CF patients.
\end{abstract}

Publication history: Republished from Current BioData's Targeted Proteins database (TPdb; http://www.targetedproteinsdb.com).

\section{Introduction}

One of the most common inherited genetic diseases is CF [1], which affects 1 in 3200 births globally, culminating in $\sim 1000$ new diagnoses annually. Due to the high frequency of $\mathrm{CF}$, the gravity of the symptoms and the resulting mortality, it is imperative that research is carried out to gain a better understanding of the disease and to develop new therapies. CF manifests due to mutation(s) in the CFTR gene, whose protein product is a cAMP-regulated $\mathrm{Cl}^{-}$ion channel belonging to the ATP binding cassette family [2]. In non-CF patients the CFTR protein is predominantly localized to the apical membrane of ciliate cells that line the lungs and gut, where it regulates $\mathrm{Cl}^{-}$ion movement across epithelia $[3,4]$. CFTR mutations that abrogate channel function inhibit trans-epithelial ion transport, which in turn leads to onset of CF symptoms such as pancreatic failure and lung disease, the greatest cause of CF patient mortality $[3,5]$.

\section{CFTR biogenesis}

CFTR is a 1480 amino acid polytopic glycomembrane protein comprised of two membrane-spanning domains 
(MSD1 and MSD2) (each containing six transmembrane domains (TMD)), two cytoplasmic nucleotide binding domains (NBD1 and NBD2) and a regulatory (R) region [3] (Figure 1). CFTR folding occurs in the ER and necessitates that the cytoplasmic domains be folded properly to ensure intramolecular interaction between MSDI and MSDII [6], ultimately resulting in the NBDs and R region forming a functional ion channel [7-9]. The NBDs of CFTR are responsible for binding and hydrolyzing ATP to enable ion channel function [2]. The co-translational folding of CFTR (A-form) is an inefficient, slow and complex process $[10,11]$ whereby the nascent polypeptide is concomitantly folded and inserted into the ER lipid bilayer [12] (Figure 2). Not surprisingly, 55-80\% of newly synthesized wild-type CFTR protein is improperly folded and targeted to the cytoplasmic proteasome for degradation in human cells [13-15], proposed to be due the complex and error prone folding process.

The folded and ER membrane-inserted CFTR, referred to as the immature B-form, leaves the ER via coat protein complex II (COPII)-coated vesicles [16,17] (Figure 2). CFTR then enters the Golgi apparatus where two of the Asn-linked glycans in the fourth extracellular loop are converted from immature high-mannose forms to mature complex oligosaccharides, creating C-form CFTR [12]. The mature CFTR protein is subsequently delivered to the plasma membrane where it functions as a $\mathrm{Cl}^{-}$ion channel. At the apical membrane, CFTR levels are regulated by subapical vesicle internalization, resulting in one of two fates: recycling to the plasma membrane or lysosomal degradation [12].

\section{CFTR mutations}

Over a thousand CF disease-related mutations have been identified to date, which yield a wide of range of defects in the CFTR protein. CF disease-related mutations are assigned into different classes depending on their molecular characteristics $[18,19]$. Class I mutants include deletions, frameshifts and non-sense mutations that result in prematurely truncated CFTR protein products, class II mutants are defective in intracellular trafficking (although they may exhibit a level of ion channel activity) and class III mutants are full-length proteins with little or no ion channel activity. Class IV mutants generally result in a less severe phenotype, as the CFTR protein only exhibits slightly reduced channel activity. Class $\mathrm{V}$ mutants proteins are functional but expressed at reduced levels, while class VI mutants are expressed at wild-type levels but exhibit decreased stability at the plasma membrane. The more

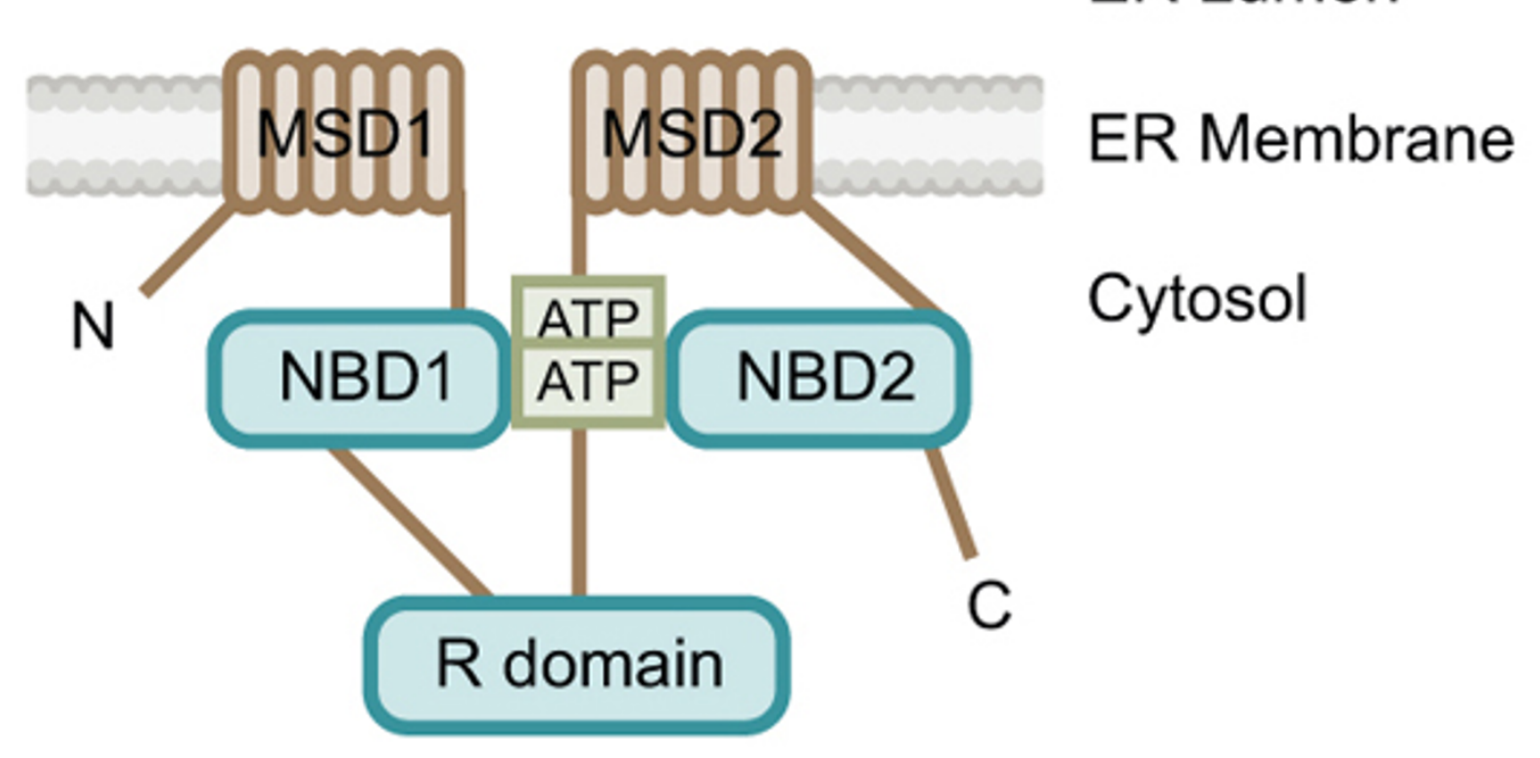

Figure I

CFTR domain layout in the ER membrane lipid bilayer during ATP hydrolysis, depicting the membrane spanning domains (MSD), nucleotide binding domains (NBD) and regulatory domain (R). The membrane spanning domains are depicted in sepia and the cytosolic domains in aqua. 


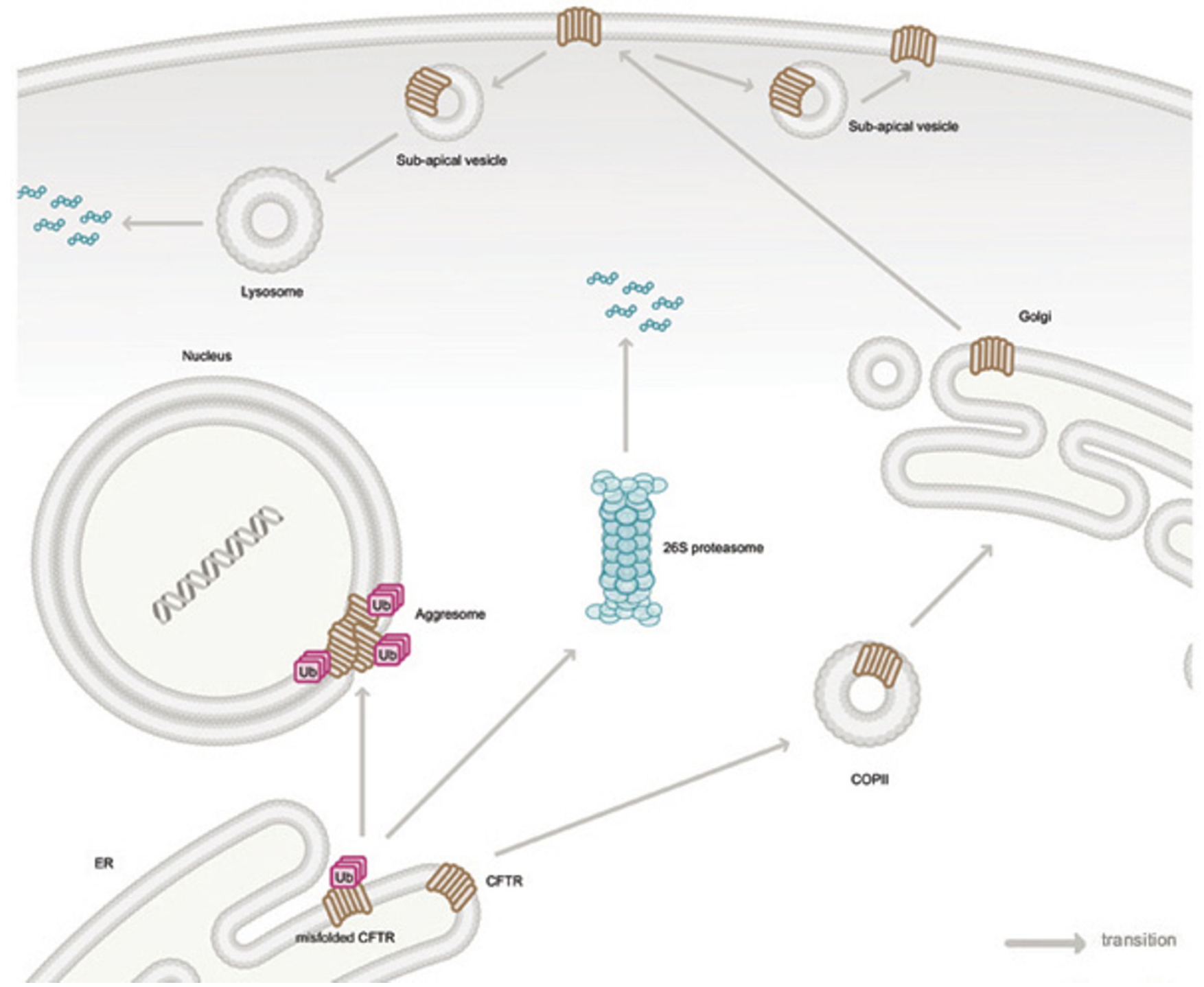

Figure 2

CFTR is co-translationally inserted into the ER membrane during ribosomal translation of CFTR mRNA from the nucleus. If CFTR is misfolded in the ER it is ubiquitylated and retrotranslocated to the cytosol, where it is degraded by the $26 \mathrm{~S}$ proteasome. Upon inhibition of the proteasome, ubiquitylated CFTR is localized to a pericentriolar aggresome structure. Correctly folded CFTR proteins are transferred to the Golgi apparatus for glycolytic maturation via the coat complex II (COPII) machinery. Mature CFTR is exported to the plasma membrane to function as a chloride ion channel. CFTR protein levels at the plasma membrane are regulated by sub-apical vesicles delivering CFTR protein for either lysosomal degradation or recycling. For simplicity, we have represented CFTR with a single membrane spanning sepia symbol. Readers are invited to refer to figures $I$ and 3 for the full domain architecture of the protein.

severe CF symptoms are associated with class I, II and III mutations due to the almost complete absence of channel activity at the plasma membrane $[18,19]$.

F508 CFTR, a temperature-sensitive class II mutation, is the most commonly identified mutation in CF patients, accounting for $70 \%$ of CF mutant alleles [20]. Other identified class II disease-causing mutations in CFTR include
N1303K, G85E and G91R [18]. These class II mutations all result in a misfolded CFTR protein that is recognized by the quality control machinery and thus prematurely degraded. The exact mechanism by which these mutations disrupt folding is not completely clear [21], but both the G85E and G91R mutations have been shown to affect folding due to the insertion of a charged residue in the plane of the lipid bilayer [9]. 


\section{The ubiquitin proteasome system (UPS)}

A cell's necessity to remove and degrade misfolded proteins directly results in the sorting of these proteins, diverting them from a folding to a degradation pathway. In addition, selecting substrates for degradation prevents their accumulation into insoluble and potentially toxic aggregates. Degradation of the misfolded proteins is carried out by the UPS, whereby substrates are polyubiquitylated and then degraded by the cytosolic proteasome [22-25]. Ubiquitylation refers to the addition of ubiquitin, a small monomeric 76 amino acid polypeptide, by covalent linkage to lysine residues on the substrate molecules [26]. Ubiquitylation is a multistep process involving three classes of enzymes: E1 ubiquitin activating enzymes, E2 ubiquitin conjugating (UBC) enzymes and E3 ubiquitin protein ligases (See [27] for review). The ubiquitin process is initiated through activation of an E1 by hydrolysis of ATP to promote the formation of a thioester bond between an internal active site cysteine and the C-terminal glycine of ubiquitin [26]. The activated ubiquitin is then transferred to the E2 active site cysteine where a new thioester linkage is formed [26]. Finally, the activated ubiquitin is covalently attached by an E3 ligase to the $\varepsilon$ amino group of a lysine side chain on the substrate protein [26]. Depending on the type of E3 involved, this step can entail an initial transfer of ubiquitin from the E2 to the E3 or the E3 can catalyze the transfer of ubiquitin directly from the E2 to the substrate protein [28]. Once a single ubiquitin molecule has been conjugated to a substrate, polyubiquitylation can occur by linking single ubiquitin molecules together to form a ubiquitin chain. Ubiquitin contains numerous lysine residues, any of which has the capacity to form isopeptide bonds. Interestingly, chains linked at lysine-63 are not targeted for degradation, whereas those linked by lysine- 29 and lysine- 48 are [29]. Ubiquitin chains vary in length and linkage [30], and their addition results in a variety of outcomes [31]. However, only polyubiquitylated proteins are targeted for 265 proteasomal degradation [29].

In the case of ER-localized proteins, polyubiquitylated substrates are dislocated from the ER membrane to enable translocation to the cytoplasmic $26 \mathrm{~S}$ proteasome, where they are selectively degraded $[24,29]$. The proteasome is a large 2.5 MDa multi-subunit complex comprising around 30 subunits [29] that degrades substrates with four or more ubiquitin molecules, with a tetra-ubiquitin motif being the minimum requirement for efficient proteasomal targeting [32].

\section{CFTR and the UPS}

Misassembled CFTR mutants appear to be detected during the folding process via two distinct systems in human cells; one that senses defects located within the cytoplasm $[21,33,34]$ and the other within the ER membrane [35]
(Figure 3). Distinct complexes of ubiquitylating proteins act in conjunction with factors such as Hsp70 and Derlin1 , which appear to recognize misassembled substrates at CFTR folding checkpoints in the cytosol and within the ER membrane, respectively.

CFTR proteins with mutations that cause misfolding of their cytoplasmic regions, (NBD1, NBD2 and the R domain) are detected by the cytosolic chaperone Hsp70. Hsp70 is believed to maintain the misfolded substrate in a soluble state, and upon interaction with the E3 ubiquitin ligase CHIP, the Hsp70-CHIP complex diverts CFTR from the folding to the degradation pathway $[33,34,36-$ 38]. CHIP promotes ubiquitylation and degradation of CFTR in association with the cytosolic E2 ubiquitin conjugating enzyme UbcH5a [33,34]. CHIP carries out this function by mediating the attachment of ubiquitin to a chaperone-presented CFTR, thereby stimulating its proteasomal degradation [33,34,36-38]. The role of CHIP in the ubiquitylation and degradation of CFTR has been demonstrated both in vitro by reconstitution of the ubiquitylation reaction [34], and in human cells by overexpression and pulse chase analysis [33].

The important role of CHIP in the degradation of CFTR is evident in studies in which CHIP's activity is inhibited by one means or another. for example, CHIP's E3 ubiquitin ligase activity can be regulated by other Hsp70 co-chaperones such as BAG-2 and HspBP1 [39-41]. BAG-2 inhibits the ubiquitin ligase activity of CHIP by abrogating the CHIP-E2 cooperation and stimulates the chaperoneassisted maturation of CFTR $[40,41]$. Likewise, overexpression of HspBP1, which has been shown to inhibit CHIP's activity and is a nucleotide exchange factor that can promote the release of substrates from Hsp70, stimulates the maturation of CFTR [39]. Furthermore, inhibition of the CHIP-Hsp70 E3 ubiquitin ligase complex by overexpression of a CHIP mutant results in the accumulation of a folding-competent stable B-form of CFTR [34]. Additionally, geldanamycin treatment of microsomes containing in vitro translated CFTR results in the release of the CFTR protein from Hsp70, which coincides with the cessation of ubiquitylation and formation of stable $\mathrm{B}$ form CFTR [42], which is resistant to ubiquitylation/degradation $[13,14,43]$.

However, while Hsp70 is necessary for the degradation of CFTR by CHIP, it also plays an important role in the folding pathway of CFTR $[7,44]$. This idea is supported by both in vitro studies in which Hsp70 can prevent the aggregation of the NBD1 domain [7], and cell culture studies which show that the induction of $\mathrm{Hsp} 70$ results in the increased trafficking of mutant $\Delta$ F508 CFTR to the plasma membrane [44]. There must exist a yet unexplained mechanism that determines whether Hsp70-bound substrates 

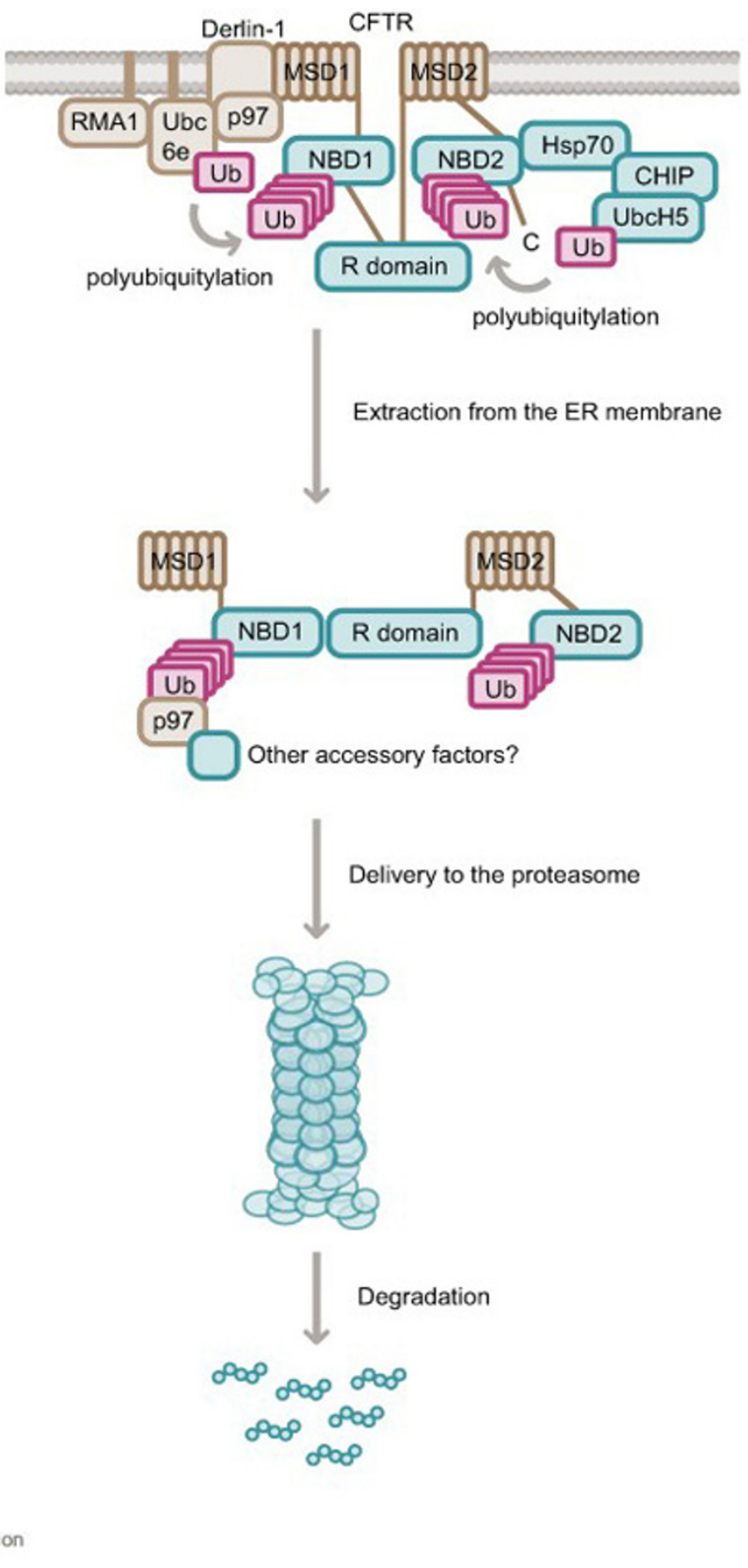

\section{Figure 3}

The UPS complexes located in the ER membrane (Derlin-I, RMAI, Ubc6e and p97) and cytoplasm (Hsp70 and CHIP) are shown. The figure demonstrates the polyubiquitylation of CFTR due to membrane-bound and cytosolic E3 ubiquitin ligase complexes. While the ubiquitylation likely occurs on the cytoplasmic domains of CFTR, the exact lysine residues conjugated with ubiquitin are unknown. $\mathrm{p} 97$ and possibly other associated factors are thought to participate in the extraction and delivery of CFTR from the ER membrane to the cytosolic proteasome. However, it is unknown whether the CFTR protein is extracted from the membrane in one piece, or degraded into smaller domains before retrotranslocation. Membrane spanning domains are depicted in sepia and the cytosolic domains in aqua. 
are allowed to fold or be targeted for degradation by the co-chaperone, CHIP.

In addition to the CHIP-Hsp70 complex, which monitors the cytosolic domains of CFTR, there are also membraneanchored proteins that can potentially monitor the assembly of CFTR membrane domains. Aberrant CFTR folding within the ER lipid bilayer is proposed to be identified by the ER quality control (QC) factor Derlin-1 and its associated proteins $[35,45]$. Co-immunoprecipitation analysis of both yeast and human cells has shown that Derlin-1 associates with substrate proteins and other QC factors such as the retro-translocation factors p97/Cdc48 and VIMP, E2 (Ubc6e) and E3 ubiquitin ligases (RMA1, HRD1 and gp78), and the deglycosylating enzyme peptide $N$ glycanase [35,45-51]. Studies in human cells have shown that the overexpression of Derlin-1 leads to the retention of CFTR in the ER $[35,45]$, while RNAi mediated knockdown of Derlin-1 leads to an increase in steady state levels of mutant CFTR [45]. These results suggest that Derlin-1 can participate in the selection of misfolded membrane proteins such as CFTR for ER-associated degradation (ERAD).

Within the context of the ER membrane, overexpression studies in human cells have shown that the E2 ubiquitin conjugating enzyme Ubc6e functions in association with the E3 ubiquitin ligase RMA1 to mediate the ubiquitylation of aberrant CFTR proteins, promoting their degradation. Both Ubc6e and RMA1 are localized to the cytosolic face of the ER membrane via their C-terminal domains [35,52-54] and therefore are likely to ubiquitylate cytosolic regions of CFTR that are exposed in its misfolded form. while both of these proteins have been isolated in complex with Derlin-1 [35], it has not been established whether or not this interaction is necessary for Ubc6e and RMA1 to promote the ubiquitylation of CFTR.

Misfolded membrane proteins that are identified by the ER QC factors are polyubiquitylated and dislocated from the ER membrane, then transported to the 26S proteasome for degradation. Yeast lacking Derlin-1 show increased stability and accumulation of ERAD substrate protein in the ER lumen, suggesting an additional function for Derlin-1 in retro-translocation [55]. Derlin-1 appears to contribute to retro-translocation of substrates such as MHC class I heavy chain molecules from the ER to the cytosol in association with p97 $[46,48,56]$. p97 is also required for mutant CFTR degradation $[57,58]$ and specifically associates with ubiquitylated CFTR proteins [45]. It acts to remove ubiquitylated CFTR from the ER to enable its degradation [59], as disruption of p97-CFTR complexes results in accumulation of immature CFTR in the ER $[60,61]$.

\section{CF disease models, knockouts and assays}

Basic CF research to understand CFTR biogenesis and degradation has been carried out in immortalized cell line systems such as HEK293 transfected with CFTR due to ease of manipulation [62]. Theses findings have subsequently been investigated in CFTR-expressing cell lines such as Fischer rat thyroid (FRT) epithelial cells [63], and in primary mouse cultures of polarized epithelial cells [64], both of which represent more biologically relevant model systems. To extend the observations made in transformed and rodent model cell lines, human bronchial epithelial (HBE) cells cultured as a monolayer have also been employed [65]. HBE cells are not used in initial studies as they are less amenable to manipulation by transfection and CFTR expression is low. However, HBE cells make excellent candidates for testing potential drug treatments.

There have been multiple attempts to create mouse models of CF that mimic the human disease (see [66] for comprehensive review). Unfortunately, there are limitations in using whole organisms such as mice for physiological studies due to differences in airway epithelial biology when compared with humans that present challenges in evaluating CF therapies using murine models [67]. Nevertheless, the models that would be of the greatest use for studies relating to the role of the UPS in CF are $\Delta \mathrm{F} 508$ models. Three such models have been generated by Doorninck et al. [68], Colledge et al. [69] and Zeiher and colleagues [70]. The models show different levels of survival and different phenotypes, likely due to the differences in mRNA and proteins levels. To date, due to the difficulties in performing in vivo studies on protein folding and degradation, these models have mainly been used to study the physiological effects of the $\Delta$ F508 mutant. However, $\triangle F 508$ CFTR exhibits a temperature sensitive folding and processing defect in epithelial cells isolated from the $\Delta$ F508 CFTR mouse [69]. Therefore, these models should prove useful in the study of the role of the UPS in $\mathrm{CF}$ and for the development of novel therapeutic approaches based on overcoming the folding and processing defect. As the list of QC factors that participate in the degradation of mutant CFTR is further developed, the CF field can also benefit from studies in which the $\Delta \mathrm{F} 508$ mouse models are crossed with knockout models of the different QC factors.

\section{Drugs with potential for CF therapeutics}

Restoration of just $5 \%$ of wild-type CFTR function dramatically improves lung and gut function in CF patients [71]. Research efforts into CF therapy development have generated drugs that can be divided into the different classes of potentiators or correctors, both of which will be discussed in more detail. In addition, the possibility of developing compounds that activate alternative $\mathrm{Cl}^{-}$and $\mathrm{K}^{+}$ 
channels to compensate for the loss of CFTR activity is promising according to studies in both HEK 293 cells and IB3-1 cells, which were isolated from a CF patient expressing the $\Delta \mathrm{F} 508$ mutation. In these studies, the cells show an increase in $\mathrm{Cl}^{-}$transport due to manipulation of $\mathrm{ClC}-2$ channels through extracellular $\mathrm{pH}$ or by arachidonic acid, amidation or acid-activated omeprazole $[72,73]$.

Potentiator drugs act to open up the malfunctioning CFTR channel, thereby promoting better ion and fluid trafficking through epithelia to relieve CF patient symptoms. This class of drugs is important for those CF mutations that result in reduced channel activity, but potentiation of channel activity alone does not solve the trafficking problems seen with the class II mutants such as $\Delta$ F508 CFTR. Alternatively, corrector molecules can help correct the folding and trafficking defect of mutant CFTRs, but they do not necessarily solve the problems of low channel activity or stability at the plasma membrane. Therefore, mutations such as F508, which exhibit defects in both folding and channel activity, will likely require combinatorial drug approaches.

Genistein [74-77] is a molecule that has been widely used in research assays as a potentiator of CFTR channel activity. It is a tyrosine kinase inhibitor but may act by binding directly to the NBDs of CFTR and stabilizing their dimerization [78]. However, like other potentiators, genistein has been shown to have a dual effect where low micromolar concentrations stimulate CFTR chloride currents, but higher concentrations inhibit CFTR channel activity [79]. CFpot-532 is another potentiator that was recently identified by Vertex Pharmaceuticals $[80,81]$; however, the mechanism of action for this drug is still unknown. The ability of CFpot-532 to act as a potentiator for mutant CFTR channels was first demonstrated with temperaturecorrected $\Delta$ F508 CFTR expressed in NIH 3T3 cells [81], and was later confirmed using low temperature rescue in BHK cells stably expressing $\triangle$ F508 CFTR [80]. This drug has exciting possibilities since studies in the BHK cells showed that it could also act as a specific corrector for CFTR and promote the maturation and trafficking of $\Delta$ F508 CFTR (but not that of a mutant P-glycoprotein) to the plasma membrane [80].

Corrector compounds aid CFTR folding [82], shielding misfolded CFTR from the UPS, which results in a greater level of CFTR protein and increased potential that it will reach the plasma membrane and provide ion channel activity. Although misfolded, certain CFTR mutants, such as F508, have residual channel activity if helped to reach the plasma membrane. It is unclear how corrector molecules function in vivo and further investigations are required to elucidate their modes of action.
Several classes of small molecule correctors have been identified, including curcumin [83], compound 9 [84], VRT-325 [81], CFpot-532 [80], Corr-3a and Corr-4a [82]. Curcumin is an ingredient in curry spice that acts as a calcium-adenosine triphosphatase pump inhibitor, and was initially identified as a compound that could correct the folding defect of $\Delta$ F508 CFTR in mice [83]. Though curcumin has been tested in phase I clinical trials, there is some controversy surrounding whether it acts as a corrector for CFTR in biological systems, as these initial results have not been reproduced by many labs [85-87]. Furthermore, both curcumin and compound 9 have been reported to be inactive in $\triangle \mathrm{F} 508 \mathrm{HBE}$ cells [81], making them unlikely therapy candidates for CF patients. The fact that these drugs were initially identified in model experimental systems, and that results could not be recapitulated in more relevant systems such as HBE cells and human patients, underscores the difficulty of developing small molecule therapeutics for CF. In terms of the current potential drug therapies for CF patients, VRT-325 (developed by Vertex Pharmaceuticals) [81], Corr-3a and Corr-4a (developed by Verkman and colleagues at University of California, San Francisco) [82] are excellent candidates. They were identified by high-throughput screens and function to increase CFTR protein levels and elevate ion channel activity. However, these drugs are still in the pre-clinical stage, and the exact mechanisms by which they function are not yet elucidated.

Application of Corr-4a or VRT-325 molecules promotes increased protein levels of $\Delta \mathrm{F} 508$ CFTR at the plasma membrane $[81,82]$. This is presumably because these correctors increase folding efficiency post-translationally, as VRT-325 promoted correct folding of mutant CFTR TMDs [88], thereby reducing ERAD and resulting in greater export of mutant CFTR to the cell surface $[81,82]$. It is not known if these compounds act directly with the CFTR protein to correct a folding defect, or if they act indirectly through other proteins or perhaps through modification of the lipid bilayer itself. However, it is known that none of these corrector molecules affect CFTR translation $[81,82]$ and that VRT-325 does not inhibit the UPS [81]. $\Delta$ F508 CFTR channel activity was increased by treatment with VRT-325 in HBE cells from CF patients and Corr-4a and Corr-3a at $37^{\circ} \mathrm{C}$ in human airway epithelial $[81,82]$. However, Corr-3a was unable to sustain $\triangle F 508$ CFTR ion channel function for longer than 24 hours [82]. The inability of theses correctors to sustain CFTR channel activity in the long term needs to be considered in terms of their potential for patient treatment. Promisingly, the activities of 'Corr' correctors are specific for $\triangle \mathrm{F} 508$ CFTR in HBE cells and did not affect the CFTR mutants P574H or N1303K, or the dopamine receptor mutant [82]. However, the activities of VRT-325 are not specific for CFTR, as these molecules also increased cell surface expression of 
the cardiac potassium channel hERG mutant G601S, which causes hereditary human long-QT syndrome type 2 [81]. Overall, the level of correction achieved with these molecules is currently low, and alternative molecules or combinations need to be developed that correct CFTR folding and activity more efficiently and effectively.

\section{Next frontiers}

Combinations of molecules for CF treatment are likely to be the most promising method of elevating CFTR protein levels and increasing ion channel activity. For example, the potentiator VRT-532 was able to potentiate ion channel activity in the CFTR mutants $\triangle F 508$ and G551D, inferring its potential as a drug treatment for CF patients in combination with VRT-325 [81]. In fact, in primary homozygous $\Delta \mathrm{F} 508 \mathrm{CF}$ airway cultures, treatment with both VRT-325 and VRT-532 increased $\Delta$ F508 CFTR maturation and resultant channel activity to levels greater than observed for each molecule independently [81].

With the identification of new CFTR degradation-associated QC factors, targeting the UPS system and enhancing ion channel activity of misfolded mutant CFTR in parallel is an optimistic avenue for current CF research. Further investigations are required to develop molecules that are specific to CFTR and that can sustain their additive effect on the patient. Many considerations must be taken into account during the development of new therapeutic molecules, such as the longevity of their effect, the effectiveness of their abilities and the specificity of their activity towards CFTR. The combination of new insights into CFTR QC factors and the UPS, and the rapid development of CF drug molecules such as correctors, has the potential to create therapies that will benefit CF patients.

\section{Abbreviations}

$\mathrm{CF}=$ Cystic Fibrosis; CFTR = Cystic Fibrosis Transmembrane Receptor; COPII = Coat Complex II; ER = Endoplasmic Reticulum; ERAD = ER-associated degradation; FRT = Fischer Rat Thyroid; HBE = Human Bronchial Epithelia; MSD = Membrane Spanning Domain; NBD = Nucleotide Binding Domain; QC = Quality Control; $\mathrm{R}=$ Regulatory; $\mathrm{TMD}=$ Transmembrane Domains; $\mathrm{UBC}=$ Ubiquitin conjugating ; UPS = Ubiquitin Proteasome System.

\section{Competing interests}

The authors declare that they have no competing interests.

\section{Acknowledgements}

Thanks to the NIH and Cystic Fibrosis Foundation for funding work in the lab of DMC, and NIGMS SPIRE postdoctoral Fellowship for funding of MFNR.

This article has been published as part of BMC Biochemistry Volume 8 Supplement I, 2007: Ubiquitin-Proteasome System in Disease Part I. The full contents of the supplement are available online at http://www.biomedcen tral.com/|47|-209|/8? issue=SI.

\section{References}

I. Rommens J.M., et al.: Identification of the cystic fibrosis gene: chromosome walking and jumping. Science 1989, 245(4922): 1059-65.

2. Riordan J.R., et al.: Identification of the cystic fibrosis gene: cloning and characterization of complementary DNA. Science 1989, 245(4922): 1066-73.

3. Sheppard D.N., et al:: The amino-terminal portion of CFTR forms a regulated Cl- channel. Cell 1994, 76(6): 1091-8.

4. Kreda S.M., et al.: Characterization of wild-type and deltaF508 cystic fibrosis transmembrane regulator in human respiratory epithelia. Mol Biol Cell 2005, 16(5):2 154-67.

5. Roomans G.M.: Pharmacological approaches to correcting the ion transport defect in cystic fibrosis. Am J Respir Med 2003, 2(5):4|3-3|.

6. Smyth S.S., Patterson C.: Tiny dancers: the integrin-growth factor nexus in angiogenic signaling. J Cell Biol 2002, I58(I): 17-2I.

7. Meacham G.C., et al.: The Hdj-2/Hsc70 chaperone pair facilitates early steps in CFTR biogenesis. Embo J 1999, 18(6): | 492-505.

8. Ostedgaard L.S., et al.: Association of domains within the cystic fibrosis transmembrane conductance regulator. Biochemistry 1997, 36(6): 1287-94.

9. Xiong $X$., et al.: Structural cues involved in endoplasmic reticulum degradation of G85E and G9 IR mutant cystic fibrosis transmembrane conductance regulator. J Clin Invest 1997, 100(5): 1079-88.

10. Patterson $C .:$ A new gun in town: the $U$ box is a ubiquitin ligase domain. SCi STKE 2002, 2002(II6):PE4.

II. Kleizen B., et al:: Folding of CFTR is predominantly cotranslational. Mol Cell 2005, 20(2):277-87.

12. Gelman M.S., Kopito R.R.: Rescuing protein conformation: prospects for pharmacological therapy in cystic fibrosis. J Clin Invest 2002, II 10(II): I591-7.

13. Lukacs G.L., et al:: Conformational maturation of CFTR but not its mutant counterpart (delta F508) occurs in the endoplasmic reticulum and requires ATP. Embo J 1994, I3(24):6076-86.

14. Ward C.L., Kopito R.R.: Intracellular turnover of cystic fibrosis transmembrane conductance regulator. Inefficient processing and rapid degradation of wild-type and mutant proteins. J Biol Chem 1994, 269(4I):25710-8.

15. Jensen T.J., et al.: Multiple proteolytic systems, including the proteasome, contribute to CFTR processing. Cell 1995, 83(I): I29-35.

16. Wang $X$., et al:: COPII-dependent export of cystic fibrosis transmembrane conductance regulator from the ER uses a di-acidic exit code. J Cell Biol 2004, I67(1):65-74.

17. Yoo J.S., et al.: Non-conventional trafficking of the cystic fibrosis transmembrane conductance regulator through the early secretory pathway. J Biol Chem 2002, 277(13): I I40 I-9.

18. Rowe S.M., Miller S., Sorscher E.J.: Cystic fibrosis. N Engl J Med 2005, 352(19): 1992-200I.

19. Rubenstein R.C.: Novel, mechanism-based therapies for cystic fibrosis. Curr Opin Pediatr 2005, I 7(3):385-92.

20. Zielenski J., Tsui L.C.: Cystic fibrosis: genotypic and phenotypic variations. Annu Rev Genet 1995, 29:777-807.

21. Cyr D.M.: Arrest of CFTRDeltaF508 folding. Nat Struct Mol Biol 2005, I 2(1):2-3.

22. Ward C.L., Omura S., Kopito R.R.: Degradation of CFTR by the ubiquitin-proteasome pathway. Cell |995, 83(I): |2|-7.

23. Johnston J.A., Ward C.L., Kopito R.R.: Aggresomes: a cellular response to misfolded proteins. J Cell Biol I 998, I43(7): I 883-98.

24. Xiong X., Chong E., Skach W.R.: Evidence that endoplasmic reticulum (ER)-associated degradation of cystic fibrosis transmembrane conductance regulator is linked to retrograde translocation from the ER membrane. J Biol Chem 1999, 274(5):2616-24.

25. Gelman M.S., Kannegaard E.S., Kopito R.R.: A principal role for the proteasome in endoplasmic reticulum-associated degradation of misfolded intracellular cystic fibrosis transmembrane conductance regulator. J Biol Chem 2002, 277( (14): I I709- I4. 
26. Ciechanover A.: The ubiquitin-proteasome pathway: on protein death and cell life. Embo J 1998, 17(24):7I5I-60.

27. Hoppe T.: Multiubiquitylation by E4 enzymes: 'one size' doesn't fit all. Trends Biochem Sci 2005, 30(4): 183-7.

28. Cyr D.M., Hohfeld J., Patterson C.: Protein quality control: Ubox-containing E3 ubiquitin ligases join the fold. Trends Biochem Sci 2002, 27(7):368-75.

29. Miller J., Gordon C.: The regulation of proteasome degradation by multi-ubiquitin chain binding proteins. FEBS Lett 2005 579(15):3224-30.

30. Hochstrasser M.: Lingering mysteries of ubiquitin-chain assembly. Cell 2006, 124(I):27-34.

3I. Welchman R.L., Gordon C., Mayer R.J.: Ubiquitin and ubiquitinlike proteins as multifunctional signals. Nat Rev Mol Cell Biol 2005, 6(8):599-609.

32. Thrower J.S., et al.: Recognition of the polyubiquitin proteolytic signal. Embo J 2000, I9(I):94-102.

33. Meacham G.C., et al.: The Hsc70 co-chaperone CHIP targets immature CFTR for proteasomal degradation. Nat Cell Biol 200I, 3(I): I00-5.

34. Younger J.M., et al.: A foldable CFTR\{Delta\}F508 biogenic intermediate accumulates upon inhibition of the Hsc70CHIP E3 ubiquitin ligase. / Cell Biol 2004, 167(6): 1075-85.

35. Younger J.M., et al:: Sequential quality-control checkpoints triage misfolded cystic fibrosis transmembrane conductance regulator. Cell 2006, I26(3):57|-82.

36. Hohfeld J., Cyr D.M., Patterson C.: From the cradle to the grave: molecular chaperones that may choose between folding and degradation. EMBO Rep 200I, 2(10):885-90.

37. McDonough H., Patterson C.: CHIP: a link between the chaperone and proteasome systems. Cell Stress Chaperones 2003, 8(4):303-8.

38. Murata S., Chiba T., Tanaka K.: CHIP: a quality-control E3 ligase collaborating with molecular chaperones. Int J Biochem Cell Biol 2003, 35(5):572-8

39. Alberti S., et al.: The cochaperone HspBPI inhibits the CHIP ubiquitin ligase and stimulates the maturation of the cystic fibrosis transmembrane conductance regulator. Mol Biol Cell 2004, I 5(9):4003-10.

40. Arndt V., et al.: BAG-2 acts as an inhibitor of the chaperoneassociated ubiquitin ligase CHIP. Mol Biol Cell 2005, I 6(I 2):589|-900

41. Dai Q., et al.: Regulation of the Cytoplasmic Quality Control Protein Degradation Pathway by BAG2. J Biol Chem 2005, 280(46):38673-3868।.

42. Fuller W., Cuthbert A.W.: Post-translational disruption of the delta $\triangle$ F508 cystic fibrosis transmembrane conductance regulator (CFTR)-molecular chaperone complex with geldanamycin stabilizes delta $\triangle$ F508 CFTR in the rabbit reticulocyte lysate. J Biol Chem 2000, 275(48):37462-8.

43. Cheng S.H., et al.: Defective intracellular transport and processing of CFTR is the molecular basis of most cystic fibrosis. Cell 1990, 63(4):827-34.

44. Choo-Kang L.R., Zeitlin P.L.: Induction of HSP70 promotes DeltaF508 CFTR trafficking. Am J Physiol Lung Cell Mol Physiol 200I, 28 I (I):L58-68.

45. Sun F., et al.: Derlin-I promotes the efficient degradation of CFTR and CFTR folding mutants. / Biol Chem 2006.

46. Ye Y., et al.: Inaugural Article: Recruitment of the p97 ATPase and ubiquitin ligases to the site of retrotranslocation at the endoplasmic reticulum membrane. Proc Natl Acad Sci U S A 2005, I 02(40): |4|32-8

47. Lilley B.N., Ploegh H.L.: Multiprotein complexes that link dislocation, ubiquitination, and extraction of misfolded proteins from the endoplasmic reticulum membrane. Proc Natl Acad Sci U S A 2005, 102(40): |4296-30I.

48. $\mathrm{Ye}$ Y., et al: A membrane protein complex mediates retrotranslocation from the ER lumen into the cytosol. Nature 2004, 429(6994):84I-7.

49. Schulze A., et al:: The Ubiquitin-domain Protein HERP forms a Complex with Components of the Endoplasmic Reticulum Associated Degradation Pathway. J Mol Biol 2005.

50. Schuberth C., Buchberger A.: Membrane-bound Ubx2 recruits Cdc48 to ubiquitin ligases and their substrates to ensure efficient ER-associated protein degradation. Nat Cell Biol 2005, 7(10):999-1006.
5I. Katiyar S., Joshi S., Lennarz W.J.: The retrotranslocation protein Derlin-I binds peptide: $\mathbf{N}$-glycanase to the endoplasmic reticulum. Mol Biol Cell 2005, I6( I 0):4584-94.

52. Matsuda N., Nakano A.: RMAI, an Arabidopsis thaliana gene whose cDNA suppresses the yeast secl 5 mutation, encodes a novel protein with a RING finger motif and a membrane anchor. Plant Cell Physiol 1998, 39(5):545-54.

53. Matsuda N., et al.: Rmal, a novel type of RING finger protein conserved from Arabidopsis to human, is a membranebound ubiquitin ligase. J Cell Sci 200 I, I I 4(Pt I0): 1949-57.

54. Lenk U., et al.: A role for mammalian Ubc6 homologues in ERassociated protein degradation. I Cell Sci 2002, II5(Pt 14):3007-14.

55. Knop M., et al.: Derl, a novel protein specifically required for endoplasmic reticulum degradation in yeast. Embo J 1996, I5(4):753-63

56. Lilley B.N., Ploegh H.L.: A membrane protein required for dislocation of misfolded proteins from the ER. Nature 2004, 429(6994):834-40.

57. Gnann A., Riordan J.R., Wolf D.H.: Cystic fibrosis transmembrane conductance regulator degradation depends on the lectins $\mathrm{Htm} / \mathrm{p} / \mathrm{EDEM}$ and the Cdc48 protein complex in yeast. Mol Biol Cell 2004, I 5(9):4I25-35.

58. Weihl C.C., et al.: Inclusion body myopathy-associated mutations in P97/VCP impair endoplasmic reticulum-associated degradation. Hum Mol Genet 2006, I 5(2): 189-99.

59. Ravid T., Kreft S.G., Hochstrasser M.: Membrane and soluble substrates of the Doa 10 ubiquitin ligase are degraded by distinct pathways. Embo / 2006, 25(3):533-43.

60. Vij N., Fang S., Zeitlin P.L.: Selective inhibition of endoplasmic reticulum-associated degradation rescues DeltaF508-cystic fibrosis transmembrane regulator and suppresses interleukin-8 levels: therapeutic implications. J Biol Chem 2006, 28।(25): 17369-78.

61. Dalal S., et al.: Distinct roles for the AAA ATPases NSF and p97 in the secretory pathway. Mol Biol Cell 2004, I 5(2):637-48.

62. Younger J.M., et al: Cystic fibrosis transmembrane conductance regulator as a model substrate to study endoplasmic reticulum protein quality control in mammalian cells. Methods Mol Biol 2005, 301:293-303.

63. Sheppard D.N., et al:: Expression of cystic fibrosis transmembrane conductance regulator in a model epithelium. $\mathrm{Am}$ Physiol I 994, 266(4 Pt I):L405-I3.

64. Davidson D.J., et al.: Murine epithelial cells: isolation and culture. I Cyst Fibros 2004, 3(Suppl 2):59-62.

65. Devor D.C., Bridges R.J., Pilewski J.M.: Pharmacological modulation of ion transport across wild-type and DeltaF508 CFTRexpressing human bronchial epithelia. Am J Physiol Cell Physiol 2000, 279(2):C46I-79.

66. Guilbault C., et al.: Cystic fibrosis mouse models. Am J Respir Cell Mol Biol 2007, 36(I): I-7.

67. Liu X., et al.: Species-specific differences in mouse and human airway epithelial biology of recombinant adeno-associated virus transduction. Am J Respir Cell Mol Biol 2006, 34(I):56-64

68. van Doorninck J.H., et al.: A mouse model for the cystic fibrosis delta $\Delta$ F508 mutation. Embo J 1995, I 4( 1 8):4403-II.

69. Colledge W.H., et al.: Generation and characterization of a delta F508 cystic fibrosis mouse model. Nat Genet 1995, I 0(4):445-52

70. Zeiher B.G., et al.: A mouse model for the delta F508 allele of cystic fibrosis. J Clin Invest 1995, 96(4):205 I-64.

7I. Dorin J.R., et al.: A demonstration using mouse models that successful gene therapy for cystic fibrosis requires only partial gene correction. Gene Ther 1996, 3(9):797-80I.

72. Schwiebert E.M., et al: Analysis of CIC-2 channels as an alternative pathway for chloride conduction in cystic fibrosis airway cells. Proc Natl Acad Sci U S A 1998, 95(7):3879-84.

73. Cuppoletti J., et al.: $\mathbf{C l C}-2 \mathbf{~ C l}$ - channels in human lung epithelia: activation by arachidonic acid, amidation, and acid-activated omeprazole. Am J Physiol Cell Physiol 200I, 28 I(I):C46-54.

74. Hwang T.C., et al:: Genistein potentiates wild-type and delta F508-CFTR channel activity. Am J Physiol 1997, 273(3 Pt I):C988-98

75. Yang I.C., et al.: Modulation of CFTR chloride channels by calyculin A and genistein. Am J Physiol I997, 272(I Pt I):CI42-55. 
76. Akiyama T., et al.: Genistein, a specific inhibitor of tyrosine-specific protein kinases. J Biol Chem 1987, 262(I 2):5592-5.

77. Illek B., et al.: cAMP-independent activation of CFTR Cl channels by the tyrosine kinase inhibitor genistein. Am J Physiol 1995, 268(4 Pt I):C886-93.

78. Moran O., Galietta L.J., Zegarra-Moran O.: Binding site of activators of the cystic fibrosis transmembrane conductance regulator in the nucleotide binding domains. Cell Mol Life Sci 2005, 62(4):446-60.

79. Moran O., Zegarra-Moran O.: A quantitative description of the activation and inhibition of CFTR by potentiators: Genistein. FEBS Lett 2005, 579(18):3979-83.

80. Wang Y., et al.: Specific rescue of cystic fibrosis transmembrane conductance regulator processing mutants using pharmacological chaperones. Mol Pharmacol 2006, 70(1):297-302.

81. Van Goor F., et al:: Rescue of DeltaF508-CFTR trafficking and gating in human cystic fibrosis airway primary cultures by small molecules. Am J Physiol Lung Cell Mol Physiol 2006, 290(6):LIII 17-30.

82. Pedemonte N., et al.: Small-molecule correctors of defective DeltaF508-CFTR cellular processing identified by highthroughput screening. J Clin Invest 2005, I I 5(9):2564-7I.

83. Egan M.E., et al.: Curcumin, a major constituent of turmeric, corrects cystic fibrosis defects. Science 2004, 304(5670):600-2.

84. Mazzei M., et al:: 2-(dialkylamino)-4H-I-benzopyran-4-one derivatives modify chloride conductance in CFTR expressing cells. Farmaco 2003, 58(9):961-70.

85. Song $Y$., et al.: Evidence against the rescue of defective DeltaF508-CFTR cellular processing by curcumin in cell culture and mouse models. I Biol Chem 2004, 279(39):40629-33.

86. Dragomir A., et al.: Curcumin does not stimulate cAMP-mediated chloride transport in cystic fibrosis airway epithelial cells. Biochem Biophys Res Commun 2004, 322(2):447-5I.

87. Loo T.W., Bartlett M.C., Clarke D.M.: Thapsigargin or curcumin does not promote maturation of processing mutants of the ABC transporters, CFTR, and P-glycoprotein. Biochem Biophys Res Commun 2004, 325(2):580-5.

88. Loo T.W., et al.: The chemical chaperone CFcor-325 repairs folding defects in the transmembrane domains of CFTR processing mutants. Biochem J 2006, 395(3):537-42.

\section{Publication history}

Republished from Current BioData's Targeted Proteins database (TPdb; http://www.targetedproteinsdb.com).
Publish with Bio Med Central and every scientist can read your work free of charge

"BioMed Central will be the most significant development for disseminating the results of biomedical research in our lifetime. "

Sir Paul Nurse, Cancer Research UK

Your research papers will be:

- available free of charge to the entire biomedical community

- peer reviewed and published immediately upon acceptance

- cited in PubMed and archived on PubMed Central

- yours - you keep the copyright
BioMedcentral 